\title{
20 años de logros y nuevos retos en el Programa Cirugía Extramuros del Instituto Jalisciense de Cirugía Reconstructiva Dr. José Guerrerosantos
}

\section{0 years of achievements and new challenges in the Extramural Surgery Program Jalisco Institute of Reconstructive Surgery Dr. José Guerrerosantos}

Luis A. GUERRERO-SILVA*, Enrique CANO-GENEL**, Agustín RIVERA-GÓMEZ***,

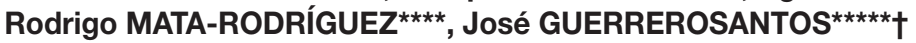

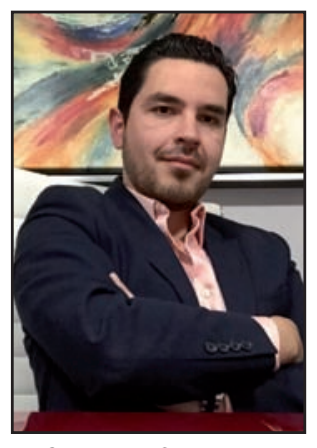

Guerrero Silva, L.A.
Resumen

Entre las múltiples estrategias del Instituto Jalisciense de Cirugía Reconstructiva Dr. José Guerrerosantos de Guadalajara (Jalisco, México) está la cobertura de servicios de salud y el brindar atención quirúrgica altruista y especializada a la población marginada del estado de Jalisco.

Recogemos en el presente trabajo los resultados de más de 20 años de esfuerzo en conjunto con el Programa de Cirugía Extramuros, que se traducen en más de 10.000 pacientes consultados y más de 6.000 cirugías realizadas en más de 100 jornadas quirúrgicas fuera del Instituto, sin importar las condiciones sociales de pobreza y las condiciones geográficas de aislamiento de las zonas donde se trabajó.

A través de este tiempo vemos como los resultados logrados implican ahora evolucionar hacia nuevas estrategias en la atención y control de calidad de los servicios quirúrgicos prestados, como gran reto a futuro para lograr soluciones quirúrgicas modernas con calidad humana y profesional.
Abstract

Among the many strategies of the Jalisco Institute of Reconstructive Surgery Dr. Jose Guerrerosantos in Guadalajara (Jalisco, México) is the coverage of health services and provide selfless and specialized surgical care to the underserved population of the state of Jalisco.

The results of more than 20 years of effort with Extramural Surgery Program, results that translate to more than 10.000 patients visited and over 6.000 surgeries performed in more than 100 surgical workshops outside the Institute, without regardless of the social conditions of poverty and geographic isolation conditions.

Through this time involving the results achieved now evolve new strategies in the care and quality control as a major challenge for the future to achieve modern surgical solutions to human and professional qualities.

\begin{tabular}{l}
$\begin{array}{l}\text { Palabras clave } \\
\text { Cirugía Plástica humanitaria, } \\
\text { Cirugía reconstructiva, Labor social. }\end{array}$ \\
$\begin{array}{l}\text { Recibido [esta versión] } \\
\text { Aceptado }\end{array}$ \\
\hline
\end{tabular}

Key words Humanitary Plastic Surgery, Reconstructive surgery, Social action.

Received [this version]

18 july/2017 Accepted

Conflicto de intereses: el autor declara no tener ningún interés financiero relacionado con el contenido de este artículo.

* Médico Residente.

** Cirujano Plástico, Jefe de Enseñanza.

*** Cirujano Plástico, Coordinador General del Programa Cirugía Extramuros.

**** Cirujano Plástico.

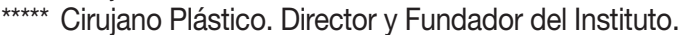

Instituto Jalisciense de Cirugía Reconstructiva Dr. José Guerrerosantos, Guadalajara, Jalisco, México. 


\section{Introducción}

A nivel nacional, en México, existe un alto porcentaje de población en condiciones de pobreza y marginación extrema que les impiden tener a su alcance los servicios de salud necesarios de acuerdo con la época actual, provocando un importante retraso en la atención y solución de múltiples problemas médico-quirúrgicos en todo el país.

En base a esta necesidad, el Dr. José Guerrerosantos (Fig. 1), fundador y director hasta su fallecimiento en noviembre de 2017 del Instituto Jalisciense de Cirugía Reconstructiva (IJCR) de Guadalajara, en el Estado de Jalisco, México, como forjador de la historia de la Cirugía Plástica no solo en nuestro país sino a nivel mundial, trazó una estrategia para ampliar la cobertura de estos servicios sanitarios y hacer efectivo el derecho a la protección de la salud de toda la población del Estado de Jalisco. Incorporó para ello al sistema de trabajo del Instituto el Programa de Cirugía Extramuros de la Secretaria de Salud, que inicialmente llevó el nombre de Programa de Rehabilitación de Labio y Paladar Hendido y "Cirujano te Visita".

Las Jornadas Médico-Quirúrgicas del Programa de Cirugía Extramuros implementadas por el Instituto Jalisciense se organizan en conjunto con el Gobierno del Estado, inspiradas en el Programa Nacional de Cirugía Extramuros que inició como Secretario de Salud a nivel nacional el Dr. Jesús Kumate en el año 1989, realizando acciones en áreas rurales y suburbanas del Estado con el fin de detectar pacientes con anomalías congénitas y a la vez organizar sesiones de cirugía en los diferentes hospitales regionales de las propias comunidades para tratarlas. ${ }^{(1,2)}$

Se trata de una labor en equipo, en su momento bajo las instrucciones directas del Dr. José Guerrerosantos como Director, y hoy en día bajo la coordinación del Dr. Enrique Cano Genel, actual Jefe del Departamento de Enseñanza, y del Dr. Agustín Rivera Gómez, líder del Programa de Cirugía Extramuros del Instituto.

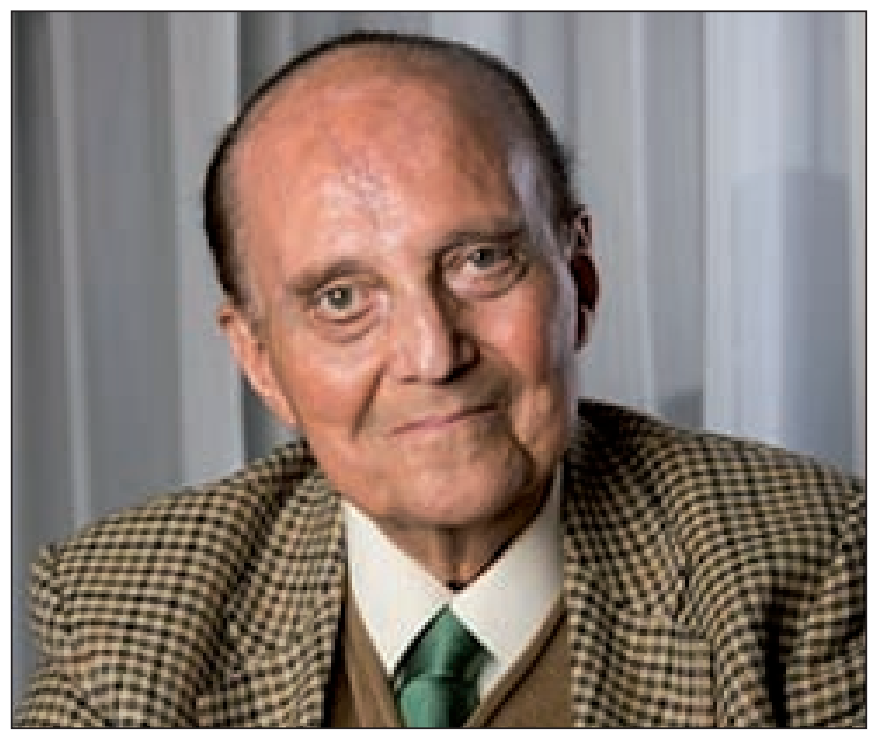

Los cirujanos del Instituto, con gran preparación y experiencia y conjuntamente con el grupo de residentes de múltiples generaciones de dicha institución, se trasladan voluntariamente a las distintas jurisdicciones de la región llevando a cabo sesiones de orientación, de consulta para diagnóstico y selección de pacientes, para posteriormente llevar a cabo las jornadas quirúrgicas en hospitales regionales de la propia Secretaria de Salud. Días después, los mismos cirujanos regresan para el seguimiento y control de los casos operados. Algunos pacientes con deformidades craneo-maxilofaciales severas son trasladados para su tratamiento quirúrgico al propio Instituto. ${ }^{(3,4)}$

Hay que reconocer que la labor del equipo de trabajo del IJCR al incorporar este programa de ayuda social, es implementarlo siempre con un amplio sentido de solidaridad, entrega y mística de servicio comunitario altruista, encauzando la motivación de servicio del personal médico y aplicando soluciones quirúrgicas modernas con calidad humana y profesional.

El objetivo de este artículo es actualizar la información de los avances logrados por el programa en el área de $\mathrm{Ci}$ rugía Reconstructiva del Instituto Jalisciense de Cirugía Reconstructiva, y los resultados obtenidos durante las últimas décadas en este campo, así como reconocer el esfuerzo enorme del equipo quirúrgico que colabora en esta labor. Al mismo tiempo, lanzar una nueva llamada a los médicos especialistas para que se sumen a las acciones resolutivas del programa en este estado y en el resto del país.

\section{Material y método}

En nuestro Programa de Cirugía Extramuros la cirugía se realiza mediante la organización de campañas quirúrgicas, eventos que se programan de forma intensiva, con 3 a 5 días de trabajo en los que se concentra a los pacientes previamente valorados y programados en el hospital sede. En dicho centro se concentran también los cirujanos, anestesiólogos, enfermeras y demás personal

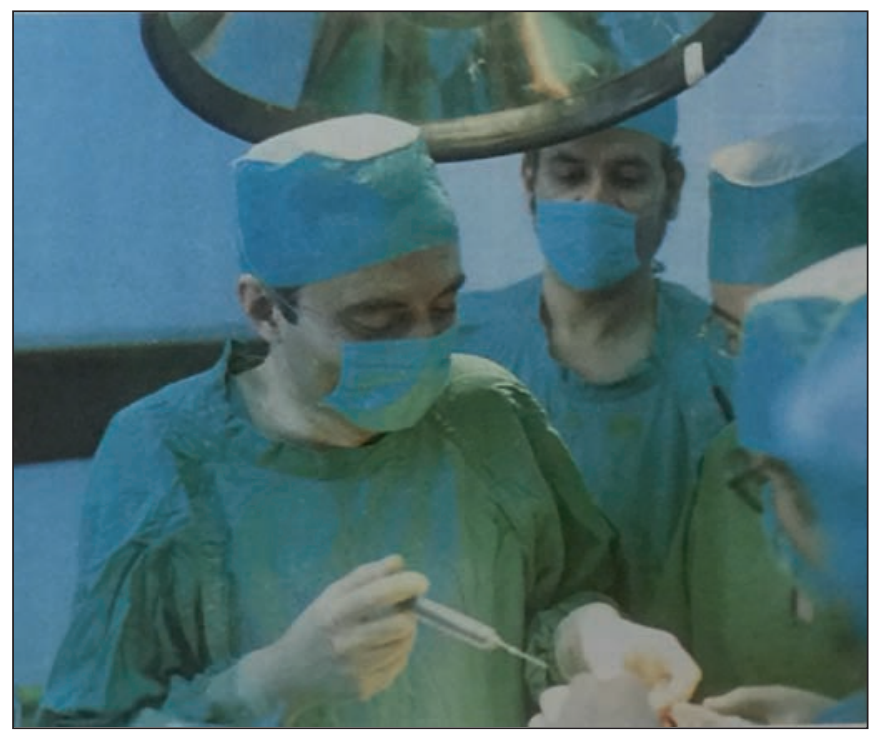

Fig. 1. Dr. José Guerrerosantos. Cirujano Plástico. Fundador y Director del Instituto Jalisciense de Cirugía Reconstructiva. 
Gráfico 1. Consultas y cirugías por año

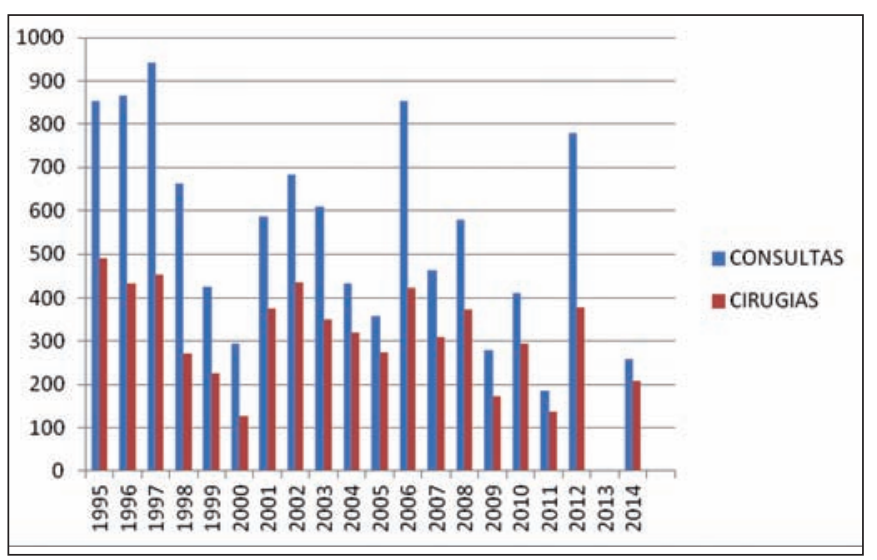

Gráfico 2. Consultas y cirugías por sede

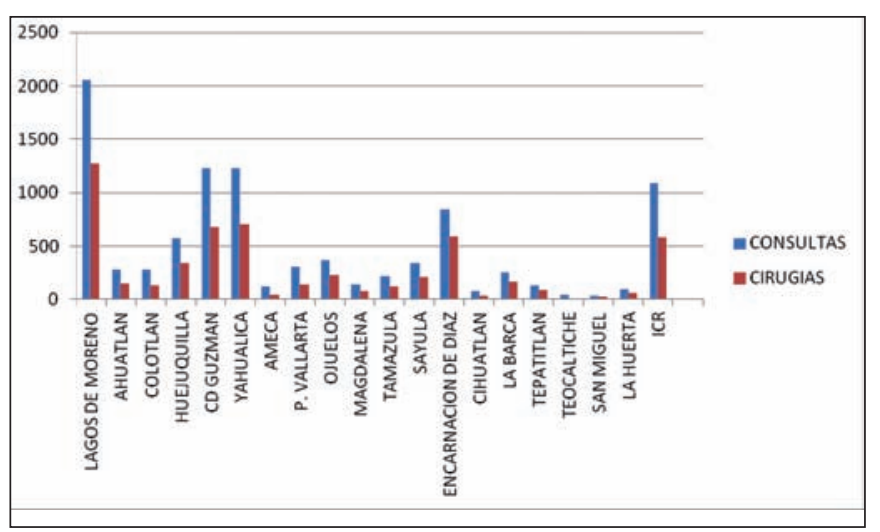

de apoyo que participará en la misión. Este personal especializado tiene un coordinador de actividades, responsable profesional directo, y que es el encargado de haber estudiado y programado a los pacientes. ${ }^{(5)}$ El grupo de trabajo definirá con los médicos del hospital sede el manejo de los pacientes y las visitas periódicas a realizar en el postoperatorio para evaluar los resultados de los procedimientos, con un total de 5 visitas a la sede por jornada. Con dicha programación se pueden recibir y administrar los insumos correspondientes para las cirugías programadas y, en caso necesario, recibir también los viáticos del personal central que se moviliza para efectuar la campaña. ${ }^{(6,7)}$

Toda esta actividad quirúrgica ha sido financiada con el presupuesto asignado al programa por parte de la Secretaria de Salud del Estado de Jalisco.

\section{RESULTADOS}

De enero de 1994 a diciembre de 2014 se organizaron, supervisaron y efectuaron 105 campañas quirúrgicas en la entidad federativa, habiendo atendido a 10485 pacientes en los que se efectuaron 6020 diferentes procedimientos quirúrgicos variados, con un promedio de 99.85 consultas y de 57.33 procedimientos por campaña; todo ello con la participación de más de 250 cirujanos (Grafico 1).

Entre las 6020 cirugías realizadas en 19 hospitales regionales del Estado de Jalisco, incluyendo cifras donde el

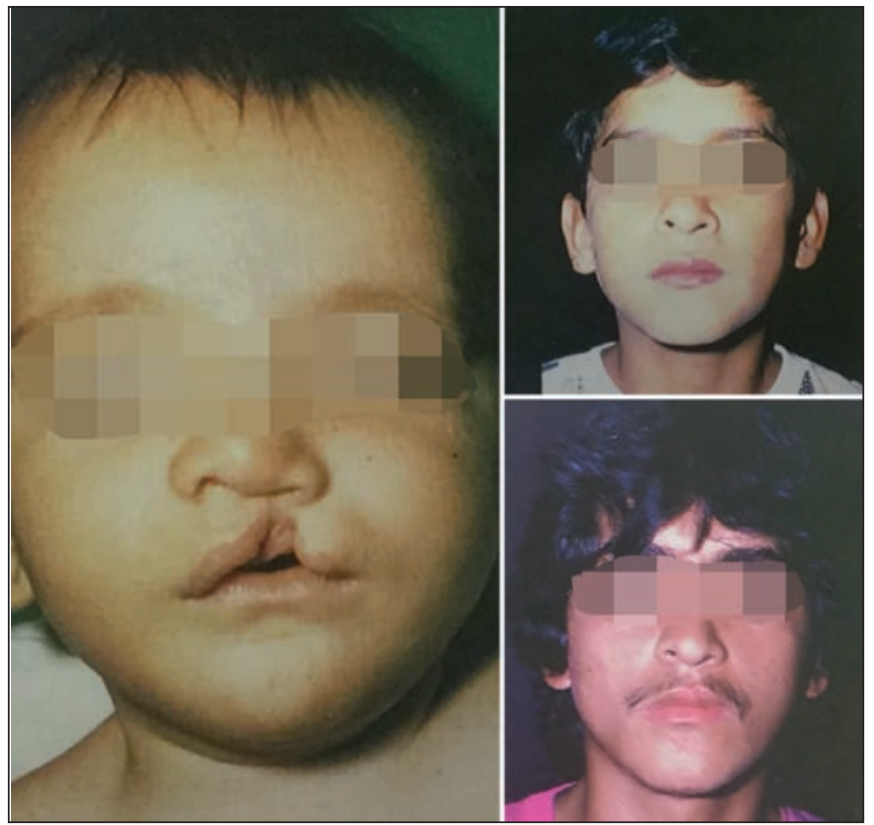

Fig. 2. Labio hendido. Evolución postquirúrgica en infancia y edad adulta.

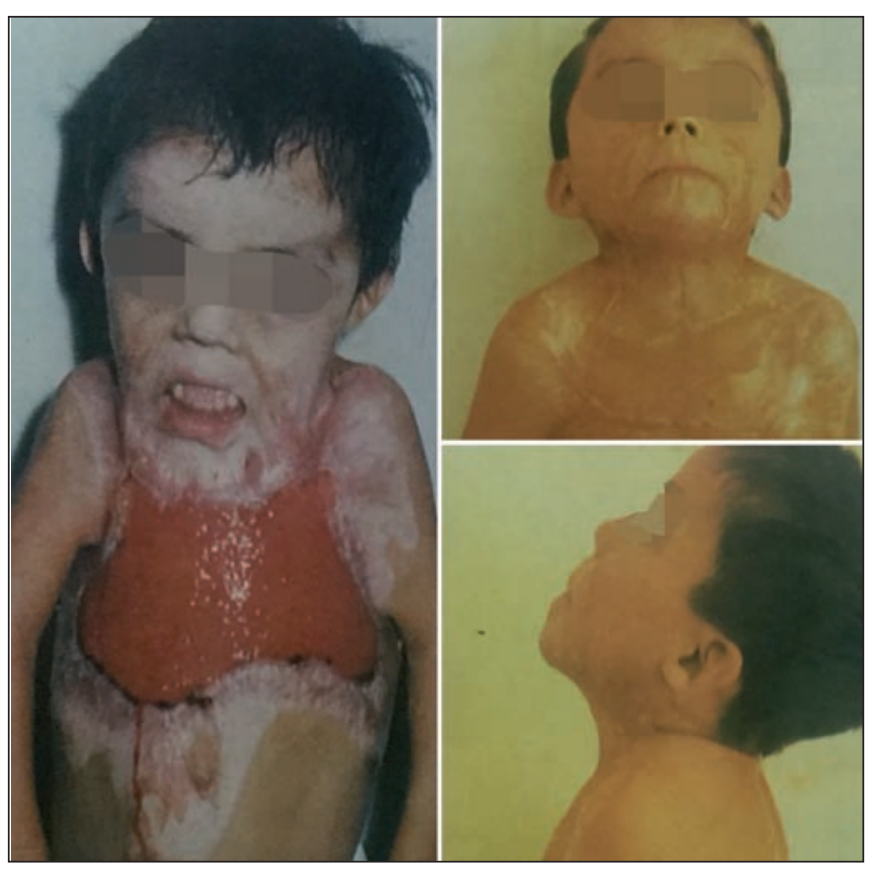

Fig. 3. Caso clínico de herida extensa en tórax y contracturas cicatriciales por secuela de quemaduras. Sinequia cervical tratada y extensión cervical corregida.

mismo Instituto Jalisciense funcionó como sede (Gráfico 2), se incluyen diversos procedimientos quirúrgicos como son; cirugía de labio y paladar hendido, cirugías craneo-maxilofaciales, cirugía de rehabilitación del paciente quemado, cirugías de secuelas de cáncer, cirugías de secuelas de traumatismo, cirugía de mano y cirugías de deformidades ósteo-musculares, por mencionar las más importantes (Fig. 2-5). Se recorrieron más de $170.000 \mathrm{~km}$ dentro del estado en las 5 visitas a cada sede por cada una de las jornadas, con distancia tanto de ida y como de regreso; lo equivalente a más de 4 vueltas a la circunferencia de la tierra por la línea ecuatorial (Tabla I).

Entre los poblados mayormente beneficiados podemos mencionar: Lagos de Moreno, Ciudad Guzmán, Ya- 


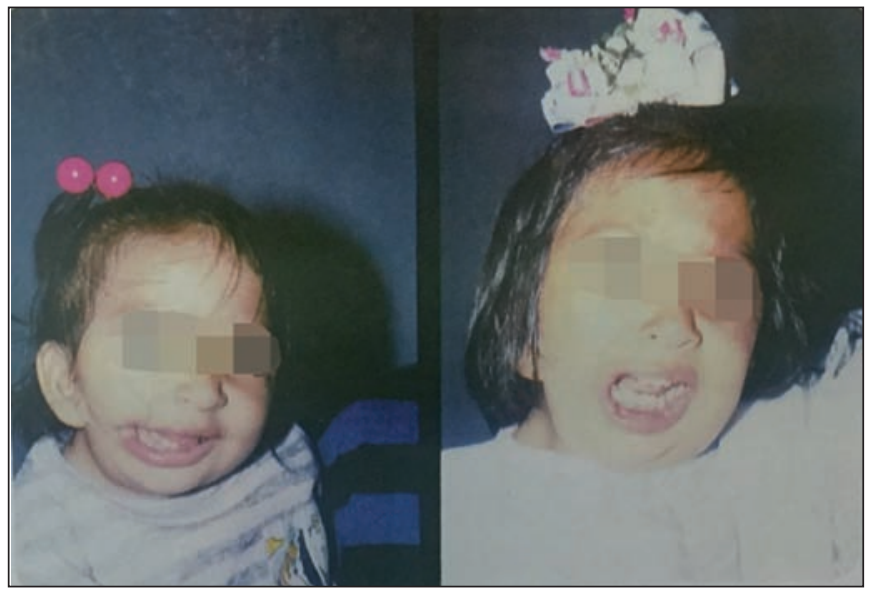

Fig. 4. Caso clínico de hendidura craneofacial tipo 7 de Tessier. Evolución postquirúrgica de la macrostomia.

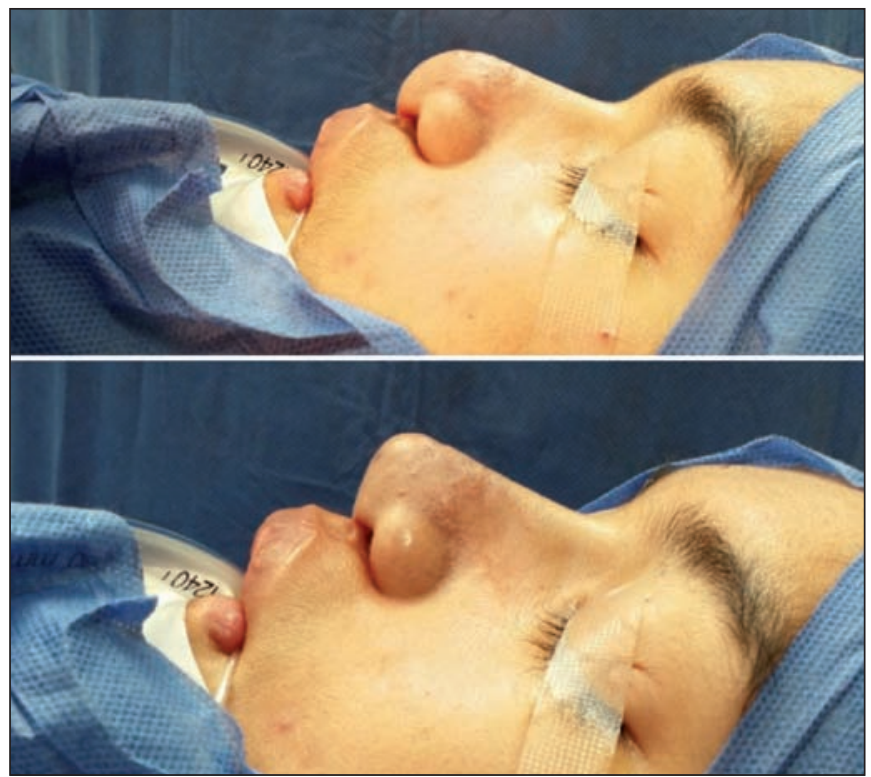

Fig. 5. Preparatoria de una joven con deformidad nasal severa por labiopaladar hendidos. Postoperatorio tras rinoplastia e injertos costales.

Tabla I. Datos generales de las campañas realizadas

\begin{tabular}{|c|c|c|c|c|c|}
\hline SEDE & CONSULTAS & CIRUGÍAS & JORNADAS & DISTANCIA km* & TOTAL de km* \\
\hline LAGOS DE MORENO & 2.053 & 1.270 & 20 & 190 & 38.000 \\
\hline AHUATLAN & 277 & 150 & 2 & 70 & 1.400 \\
\hline COLOTLAN & 279 & 133 & 5 & 206 & 10.300 \\
\hline HUEJUQUILLA & 576 & 341 & 7 & 334 & 23.380 \\
\hline CD GUZMAN & 1.226 & 678 & 15 & 131 & 19.650 \\
\hline YAHUALICA & 1.228 & 704 & 14 & 130 & 18.200 \\
\hline AMECA & 121 & 47 & 1 & 87 & 870 \\
\hline P. VALLARTA & 306 & 138 & 2 & 311 & 6.220 \\
\hline OJUELOS & 368 & 226 & 4 & 272 & 10.880 \\
\hline MAGDALENA & 141 & 78 & 2 & 88 & 1.760 \\
\hline TAMAZULA & 220 & 122 & 2 & 163 & 3.260 \\
\hline SAYULA & 337 & 205 & 4 & 118 & 4.720 \\
\hline ENCARNACION DE DIAZ & 847 & 590 & 11 & 203 & 22.330 \\
\hline CIHUATLAN & 78 & 30 & 1 & 315 & 3.150 \\
\hline LA BARCA & 256 & 166 & 3 & 101 & 3.030 \\
\hline TEPATITLAN & 126 & 88 & 2 & 75 & 1.500 \\
\hline TEOCALTICHE & 44 & 7 & 1 & 166 & 1.660 \\
\hline SAN MIGUEL & 30 & 23 & 1 & 136 & 1.360 \\
\hline LA HUERTA & 92 & 61 & 2 & 255 & 5.100 \\
\hline ICR & 1.085 & 587 & 6 & - & - \\
\hline TOTAL & 10.485 & 6.020 & 105 & & 176.770 \\
\hline
\end{tabular}


Gráfico 3. Jornadas por sede
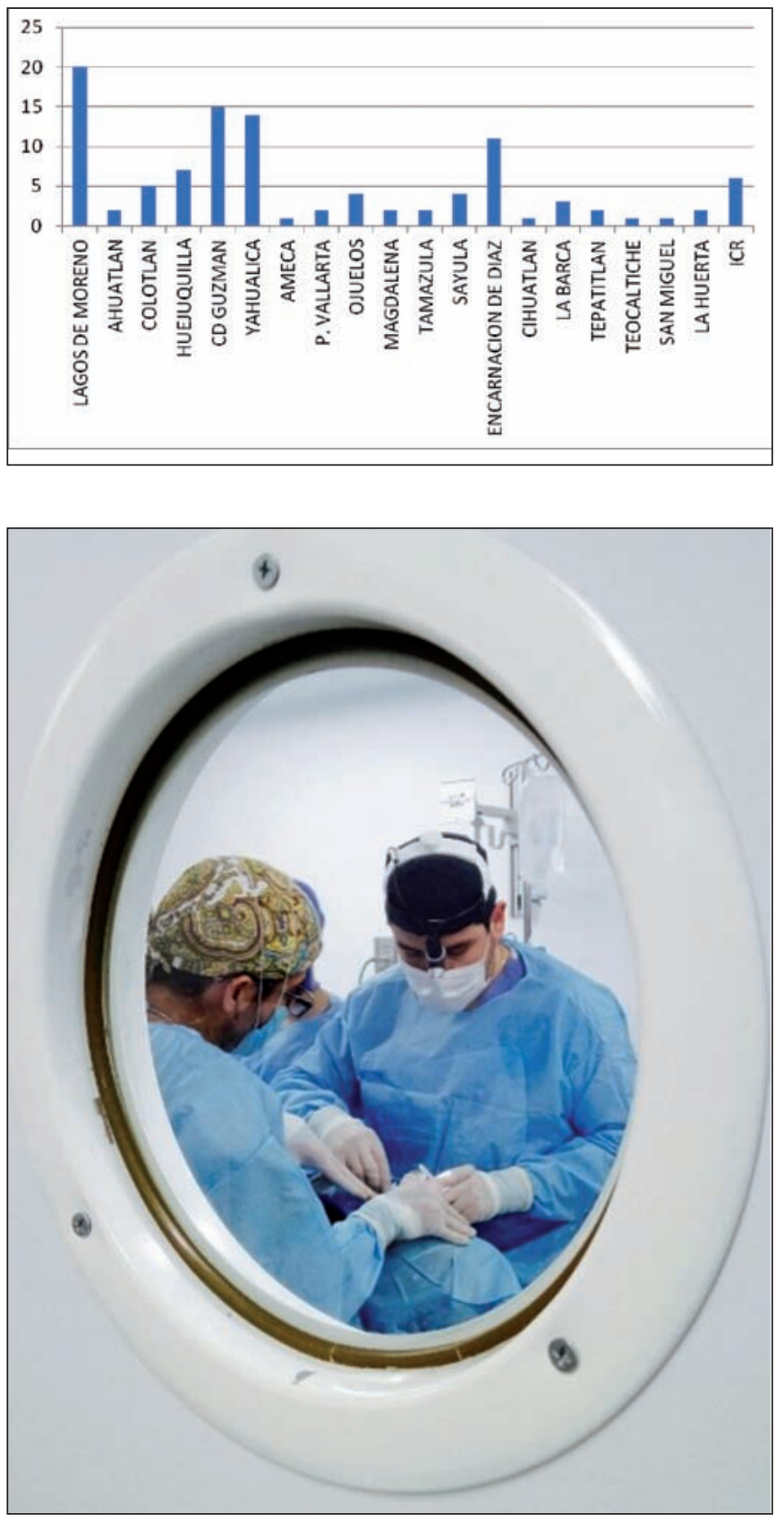

Fig. 6. Equipo quirúrgico durante la Jornada Cirujano te Visita, año 2015.

hualica y Encarnación de Díaz, con 20, 15, 14 y 11 jornadas respectivamente; en el resto de 14 poblados se llevaron a cabo menos de 10 jornadas en los 20 años del programa (Grafico 3).

Se considera a la Cirugía Plástica y Reconstructiva, una especialidad pionera en estas actividades del Programa Nacional de Cirugía Extramuros en México, con procedimientos fundamentalmente de cirugía de labio y paladar hendidos y de secuelas de quemaduras principalmente. Con el paso de los años, esta especialidad ha incrementado su participación, aunque anteriormente ésta no se llevaba a cabo en combinación con el Programa de forma directa.

Cabe destacar que la calidad de los servicios otorgados siempre está estrechamente supervisada en lo que se refiere a calidad, tanto profesional como humana, a fin

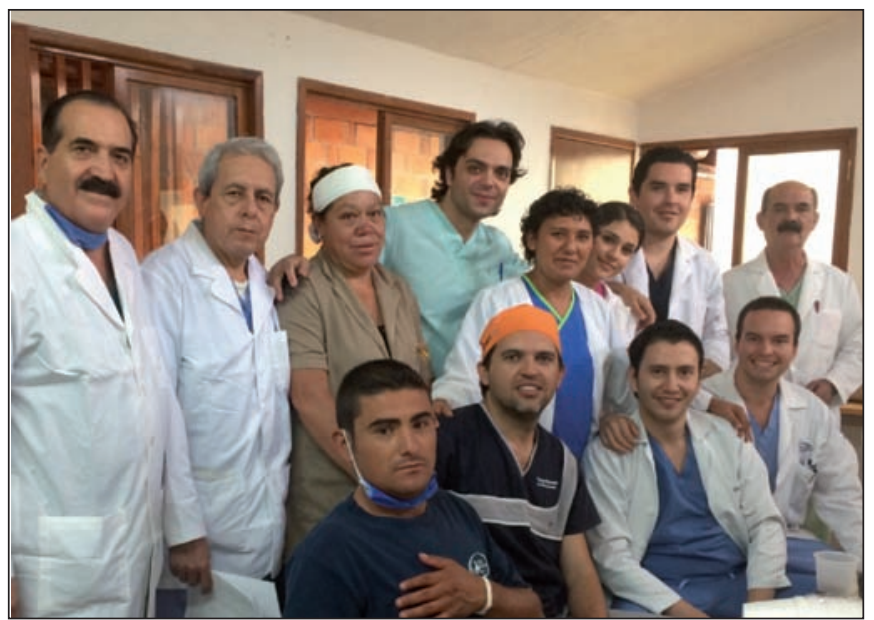

Fig. 7. Cirujanos y pacientes durante la Jornada Quirúrgica en Encarnación de Diaz, Jalisco, año 2014. Arriba: Dr. Héctor Mora Herrera (Director del Hospital de primer contacto de Encarnación de Díaz); Dr. Agustín Rivera Gómez (Coordinador General); paciente beneficiado; Dr. Marco Galati (Italia); personal voluntario; Dra. Karen Ruvalcaba (México); Dr. Luis Alberto Guerrero Silva (México); Dr. Héctor Navarrete (Secretaria de Salud Jalisco). Abajo: paciente beneficiado; Dr. Rodrigo Mata González (México); Dr. Carlos Oñate (Ecuador); Dr. Otto R. Ziegler (Perú).

de otorgar siempre a los beneficiarios un servicio de excelencia. Las complicaciones que encontramos fueron mínimas y siempre resueltas o en su propio ámbito o en algunos casos raros, trasladando a los pacientes y a sus familiares a centros de atención de tercer nivel.

\section{Discusión}

Debemos destacar el noble esfuerzo del equipo quirúrgico del IJCR (Fig. 6 y 7), de su líder y su coordinador, por la experiencia quirúrgica y el esfuerzo aportados en apoyo a esta gran labor asistencial, y sobre todo por dar respuesta a las necesidades de la población que vive en zonas de alta marginación desde las que no puedan tener fácil acceso a los servicios de cirugía reconstructiva. Existen múltiples casos y testimonios de los propios pacientes que avalan esta importante labor realizada.

Hablar de resultados y estadísticas no representa la realidad de los beneficios proporcionados por el programa. Estos beneficios se ven reflejados y manifestados por los innumerables testimonios y muestras de agradecimiento que conllevan la mejora en la calidad de vida de los pacientes beneficiados y de sus familiares, contribuyendo exitosamente para que muchos mexicanos puedan lograr así una integración óptima en la sociedad.

Es importante resaltar lo que el IJCR y el Programa Cirugía Extramuros han hecho y seguirán logrando; hacer hincapié en la necesidad de cuidar al máximo la calidad de las prestaciones otorgadas, así como de los cirujanos involucrados y su responsabilidad compartida con el resto del personal participante en el cuidado de los pacientes a todos los niveles. ${ }^{(8)}$ Es incuestionable reconocer el desempeño de los cirujanos del Instituto, de los médicos residentes, anestesiólogos, médicos ayudantes, directivos y administrativos de cada hospital como parte 
fundamental para el desarrollo de la cirugía, la importante labor desempeñada por las enfermeras instrumentistas y circulantes de cada quirófano, del personal de recuperación postoperatoria y de las centrales de equipos que preparan los requerimientos, los insumos listos y los equipos disponibles en cada momento. Sin olvidar a los trabajadores sociales y comunitarias, cuya labor es también destacada para otorgar a los pacientes los servicios comprometidos.

En la actualidad, nuestro país continúa sufriendo un retraso en la atención médica nacional, motivo por el cual siguen siendo necesarias acciones resolutivas más intensas, independientemente de las condiciones socioeconómicas de los afectados. Por esta razón, desde el IJCR se continuarán realizado esfuerzos para ampliar la cobertura de los servicios de salud a los rincones más apartados del ámbito rural del estado y del país, siempre con los más altos niveles de calidad por los que se ha caracterizado el Instituto Jalisciense de Cirugía Reconstructiva Dr. José Guerrerosantos y de esta manera continuar con las encomiendas y enseñanzas de nuestro querido maestro el Dr. José Guerrerosantos. Todavía hay mucho por hacer; consideramos la participación de todos como un privilegio de servir y un alto compromiso con el bienestar de los pacientes.

\section{In memoriam}

"Yo sigo ayudando en lo que se puede, tuve que dejar de operar y aunque extraño mucho el quirófano nunca he pensado en retirarme, la gente se retira por lo regular a los 60, mucha gente me pregunta cuándo me voy a retirar, mi única respuesta es: 15 minutos antes de que Dios me lleve al cielo"

\section{Dr. José Guerrerosantos}

(26 febrero 1932 - 20 noviembre 2017)

\section{Dirección del autor}

Dr. Luis Alberto Guerrero Silva.

Instituto Jalisciense de Cirugía Reconstructiva

Calzada del Federalismo Nte 2022

44220 Guadalajara

Jalisco, México

Correo electrónico: drgrosurgery@gmail.com

Bibliografía

1. Convivencias Quirúrgicas 1983-93. Academia Mexicana de Cirugía 1994. México.

2. Convivencias Quirúrgicas 1993-94. Academia Mexicana de Cirugía 1995. México.

3. Convivencias Quirúrgicas 1999-2000. Academia Mexicana de Cirugía. México, 2000.

4. Trigos, I. Memoria de una experiencia de atención especializada a la Salud en el medio rural 1994-2000. El Programa de Cirugía Extramuros SSA, Secretaría de Salud, México, 2000.

5. Lineamientos de operación. Programa de Cirugía Extramuros. Cuadernos técnicos SSA. Ed. Grafik, México, 2000.

6. Programa de Cirugía Extramuros. Lineamientos de Operación. Cuadernos Técnicos de la SSA. Ed. Grafik, México, 2000.

7. Reunión Nacional de Responsables Estatales de los programas y estrategias de Extensión de Cobertura, SSA, México, Junio 2000.

8. http://es.scribd.com/doc/92514770/Libro-de-CirugiaExtramuros 